\title{
Impact of postprocedural permanent pacemaker implantation on clinical outcomes after transcatheter aortic valve replacement: a systematic review and meta-analysis
}

\author{
Ziwei Xi, Tong Liu, Jing Liang, Yu-Jie Zhou, Wei Liu \\ Department of Cardiology, Beijing Anzhen hospital, Capital Medical University, Beijing Institute of Heart Lung and Blood Vessel Diseases, Beijing \\ 100029, China \\ Contributions: (I) Conception and design: Z Xi, W Liu; (II) Administrative support: Y Zhou; (III) Provision of study materials or patients: T Liu, \\ J Liang; (IV) Collection and assembly of data: Z Xi; (V) Data analysis and interpretation: Z Xi; (VI) Manuscript writing: All authors; (VII) Final \\ approval of manuscript: All authors. \\ Correspondence to: Wei Liu, MD. Department of Cardiology, Beijing Anzhen hospital, Capital Medical University, Beijing Institute of Heart Lung and \\ Blood Vessel Diseases, Anzhen Road, Chaoyang District, Beijing 100029, China. Email: liuwei@ccmu.edu.cn.
}

\begin{abstract}
Background: The incidence of conduction disturbances requiring permanent pacemaker (PPM) implantation following transcatheter aortic valve replacement (TAVR) have remained a common concern. The purpose of this study was to evaluate the impact of postprocedural PPM implantation following TAVR on clinical outcomes.

Methods: We performed a systematic search in PubMed and EMBASE databases for studies that reported raw data on clinical outcomes of patients with and without PPM implantation after TAVR and followed up patients for 10 months or longer. The primary endpoint was all-cause death. The secondary endpoints were cardiovascular death, heart failure and a composite of stroke and myocardial infarction (MI).

Results: Data from 20 studies with a total of 21,666 patients undergoing TAVR, of whom 12.5\% required PPM implantation after intervention, were analysed and the mean duration follow-up was 16.9 months. The rate of PPM ranged from $6.2 \%$ to $32.8 \%$ among different studies. A total of $6,753(31.2 \%)$ patients underwent TAVR with self-expandable prosthesis and 14,913 (68.8\%) with balloon-expandable prosthesis. The incidence of postprocedural PPM implantation was higher with the self-expandable prosthesis $(\mathrm{n}=1,717$, $25.4 \%$ ) compared with the balloon-expandable prosthesis ( $=996,6.7 \%)$. PPM after TAVR was associated with a higher risk of all-cause death (RR: 1.13; 95\% CI: 1.01-1.25; P=0.03) but not incidence of stroke and MI (RR: 0.85; 95\% CI: 0.64-1.13; P=0.27).

Conclusions: In patients undergoing TAVR, the PPM implantation after intervention was associated higher all-cause mortality but not cardiovascular mortality, heart failure and stroke or MI, which remain an unsolved issue of TAVR.
\end{abstract}

Keywords: Permanent pacemaker (PPM); transcatheter aortic valve replacement (TAVR); conduction disturbances

Submitted Jul 06, 2019. Accepted for publication Nov 19, 2019.

doi: $10.21037 /$ jtd.2019.12.02

View this article at: http://dx.doi.org/10.21037/jtd.2019.12.02

\section{Introduction}

Transcatheter aortic valve replacement (TAVR) is a less invasive treatment option for severe symptomatic aortic stenosis, and has emerged as an alternative therapeutic approach to conventional surgical aortic valve replacement for patients deemed to be at high risk of surgical complications $(1,2)$. It has been proposed that the indications of TAVR should be expanded to include patients with intermediate and even low risk of surgical complications (3-5). However, TAVR still frequently results 
in short-term complications, including cardiac conduction abnormalities and TAVR-related requirement for permanent pacemaker (PPM) implantation (6). Although technological developments in TAVR have facilitated the procedure and reduced the occurrence of some periprocedural complications, the incidence of postprocedural PPM implantation has not changed significantly over time, and has even potentially slightly increased after the introduction of newer generation devices (7).

The factors associated with conduction abnormalities and PPM implantation after TAVR have been well described, including pre-existing right bundle branch block (BBB), left BBB, use of a self-expanding bioprosthesis, and valve implantation depth (8-10). Nevertheless, it remains controversial whether PPM implantation after TAVR has a negative impact on clinical outcomes (11). Data from studies evaluating the outcome of PPM implantation following TAVR have yielded conflicting results, and several related major studies have been published in recent years. The aim of the present meta-analysis was to investigate the impact of postprocedural PPM implantation on the long-term clinical outcomes of patients who have undergone TAVR.

\section{Methods}

The present study was performed in accordance with the Preferred Reporting Items for Systematic reviews and Meta-Analyses (PRISMA) guidelines (12).

\section{Search strategy}

A literature search of the PubMed and Embase databases was performed to identify all studies published from January 1, 2002 to April 4, 2018 that investigated the impact of postprocedural PPM implantation on clinical outcomes after TAVR. The search start date was set at 2002, as that was the year when TAVR was first performed in humans. The search terms were: (TAVR OR TAVI OR percutaneous aortic valve replacement OR percutaneous aortic valve implantation) AND (pacemaker implantation OR PPM implantation OR pacing OR cardiac conduction abnormalities). The publication language was restricted to English.

\section{Study selection}

The titles and abstracts of all retrieved articles were independently reviewed by two researchers (ZW Xi, W
Liu) to identify potentially relevant studies. The full texts of all potentially relevant articles were then reviewed in detail to assess their appropriateness for inclusion in the present study. Studies fulfilling the following criteria were included: (I) data reported on long-term clinical outcomes after TAVR (follow-up $\geq 6$ months); (II) sufficient data available on postprocedural PPM implantation following TAVR. Studies were excluded if any of the following criteria applied: (I) PPM was implanted $>30$ days after TAVR; (II) abstracts, case reports, conference presentations, or editorials; (III) outcomes of interest were not clearly reported or were impossible to extract or calculate from the published results. If more than one study was published by the same authors using the same case series or overlapping case series, the study with the largest sample size was included, except when different subgroup analysis could be done. Any disagreement between the two researchers was reviewed by a third researcher (XH Chen) and resolved by consensus.

\section{Data extraction and quality assessment}

Data on clinical outcomes and the characteristics of each study were extracted using a prepared standardized extraction form. The relevant information regarding the following items were extracted by two main researchers (ZW Xi, W Liu) from each included study if available: first author's name, publication year, study period, region, study design, single or multicenter study, number of TAVR cases, patient age, proportion of males, logistic European System for Cardiac Operative Risk Evaluation, Society of Thoracic Surgeons score, valve type, follow-up duration, incidence of PPM implantation, and primary and secondary endpoints. The quality of cohort studies was evaluated using the Newcastle-Ottawa scale for prespecified items that comprised patient selection (representativeness and selection of patients, ascertainment of exposure), comparability of cohorts on the basis of design or analysis, and outcome (assessment of outcomes, adequacy of followup) (13). A quality score [0-9] was generated in accordance with a maximum score of 1 for each item.

\section{Study endpoints}

The primary endpoint was long-term (at least 10 months postoperatively) all-cause death. The secondary endpoints were cardiovascular death, heart failure, and a composite of stroke and myocardial infarction (MI). If endpoints were 


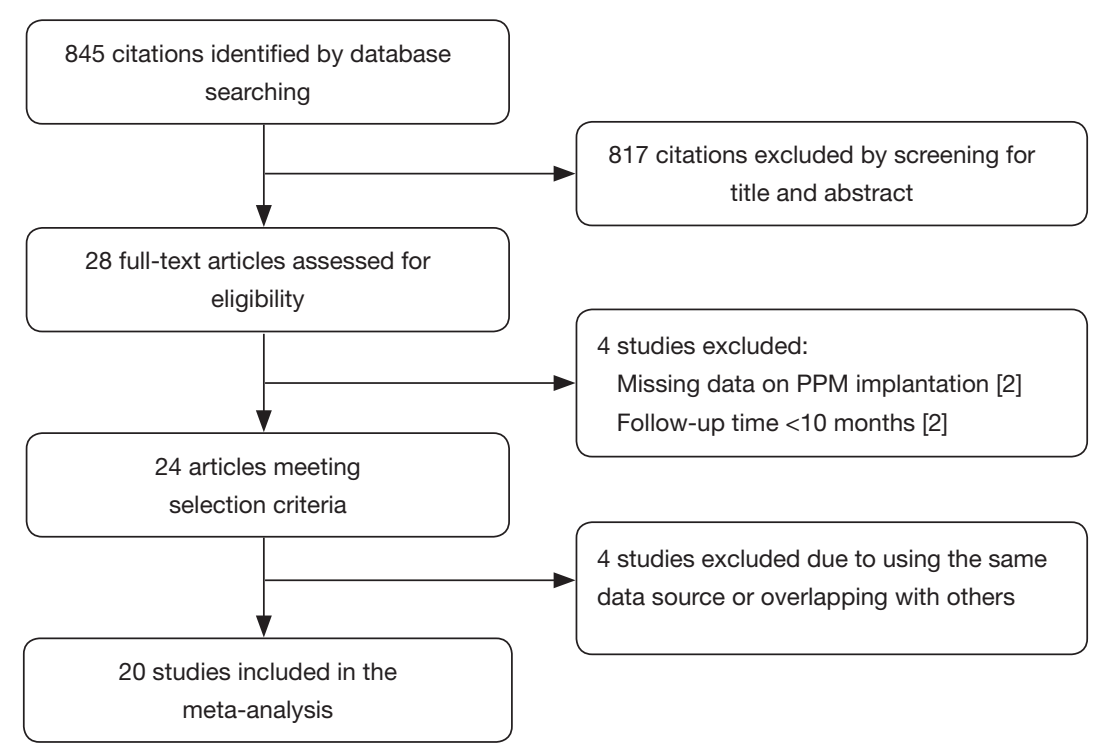

Figure 1 Flow diagram of selected studies based on the Preferred Reported Items for Systematic Reviews and Meta-Analysis (PRISMA) statement.

reported at more than one follow-up timepoint, we used data from the longest follow-up in each study.

\section{Statistical analysis}

Crude risk ratio (RR) was the principal summary measure. RRs were retrieved or directly calculated with the corresponding $95 \%$ confidence interval (CI) for each endpoint as the result of primary analysis. Heterogeneity, which was anticipated to be significant, was assessed by the Q-statistic and Higgins' and Thompson's I ${ }^{2}$ test. Significant heterogeneity was considered to be present when $\mathrm{P}<0.10$ or $\mathrm{I}^{2}>50 \%$. When there was no significant heterogeneity, the fixed-effects model (Mantel-Haenszel) was selected for the calculation of RRs, otherwise the random-effects model was selected. Sensitivity analysis was performed by deleting one study at a time and calculating the odds ratio for the remaining studies. To assess the potential effect of publication bias, we inspected funnel plots for asymmetry and used the Egger's regression asymmetry test in which $\mathrm{P}<0.10$ was considered to indicate significant publication bias. Descriptive characteristics were presented as the mean \pm standard deviation or the median (interquartile range) for continuous variables, and as percentages or frequencies for categorical variables. Data analyses were performed using the statistical package STATA V14.0 (StataCorp, College Station, Texas, USA) and Review Manager V5.3 (The
Nordic Cochrane Centre, The Cochrane Collaboration, London, UK).

\section{Results}

The literature search identified 703 articles from the PubMed database, and 272 from the Embase database; after the exclusion of duplicates, the titles and abstracts of 845 articles were reviewed. Of those, the full texts of 28 studies were assessed for eligibility. Four studies were excluded due to the use of the same data source or overlapping data with other studies. Two studies reporting different outcomes based on the presence of new-onset left BBB after TAVR were excluded due to the absence of data on PPM implantation. Another two studies were excluded due to a follow-up duration of $<6$ months. A final total of 20 studies with a total of 21,666 patients undergoing TAVR were included for the assessment of the primary endpoint; of those, seven studies were eligible for assessment of the endpoint of cardiovascular death after PPM implantation, while three studies with 12,063 patients were eligible for assessment of the endpoint of stroke and MI. The followup duration ranged from 8 to 48 months. None of the included studies were randomized. The overall quality of the included studies was good. Figure 1 shows the PRISMA flow diagram, and Table 1 shows the baseline characteristics of included studies $(8,10,14-31)$. 
$\stackrel{\circ}{2}$

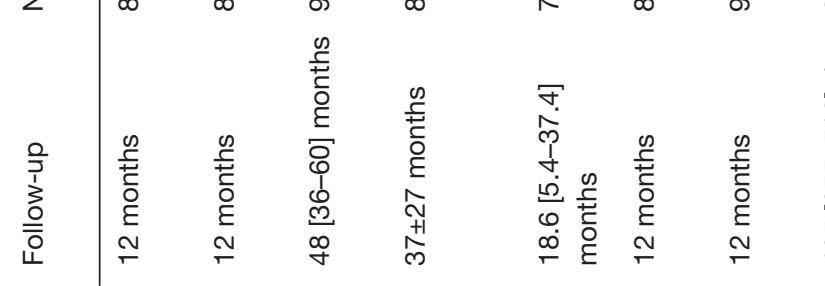

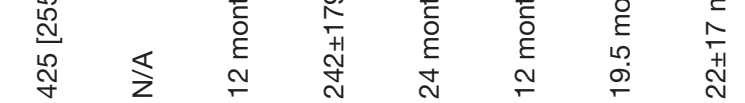

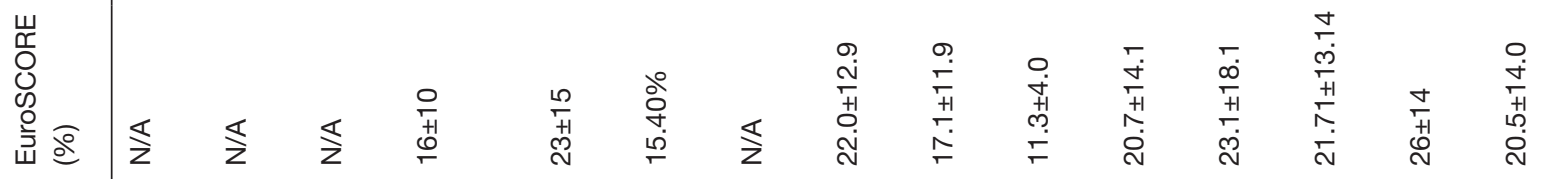

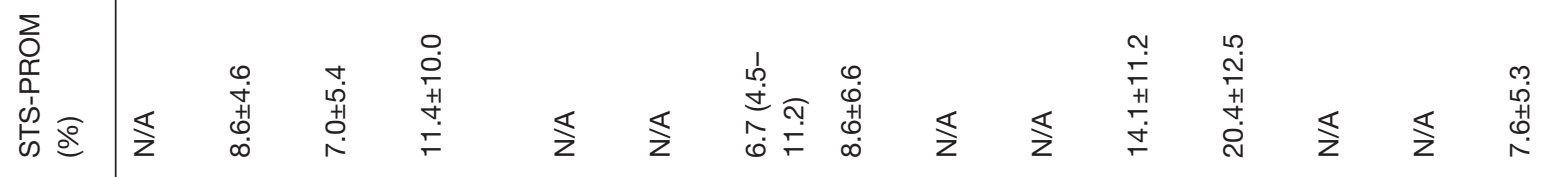

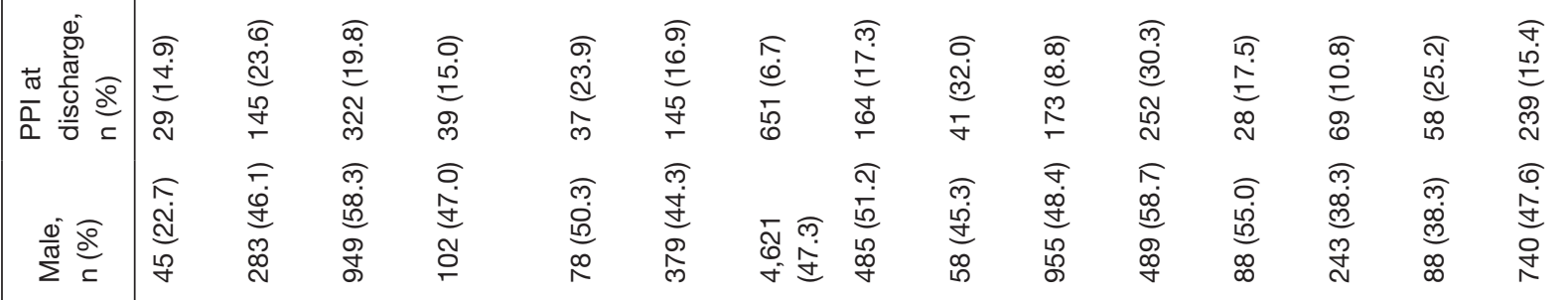

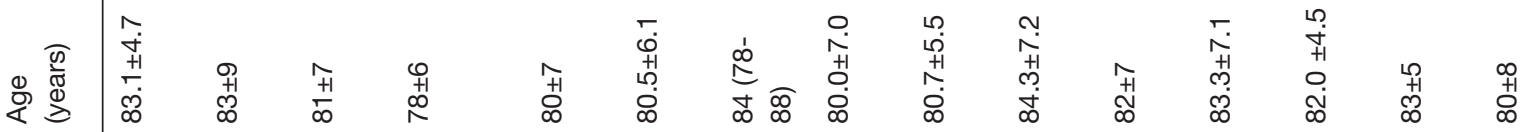

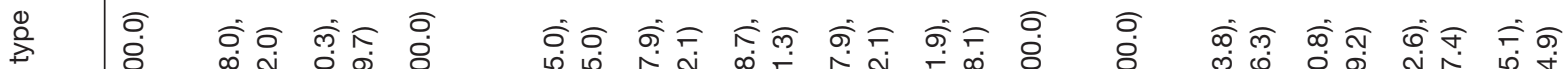

焉

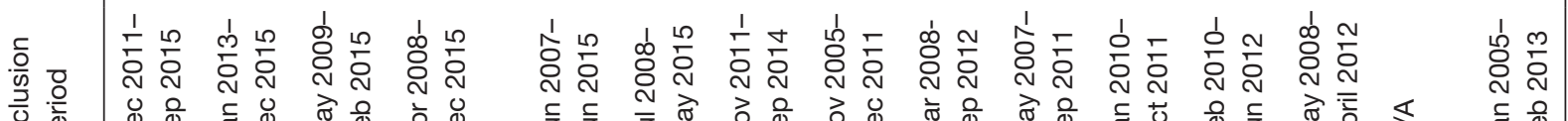

表范

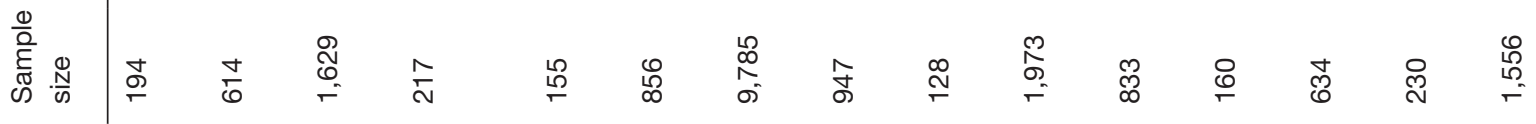

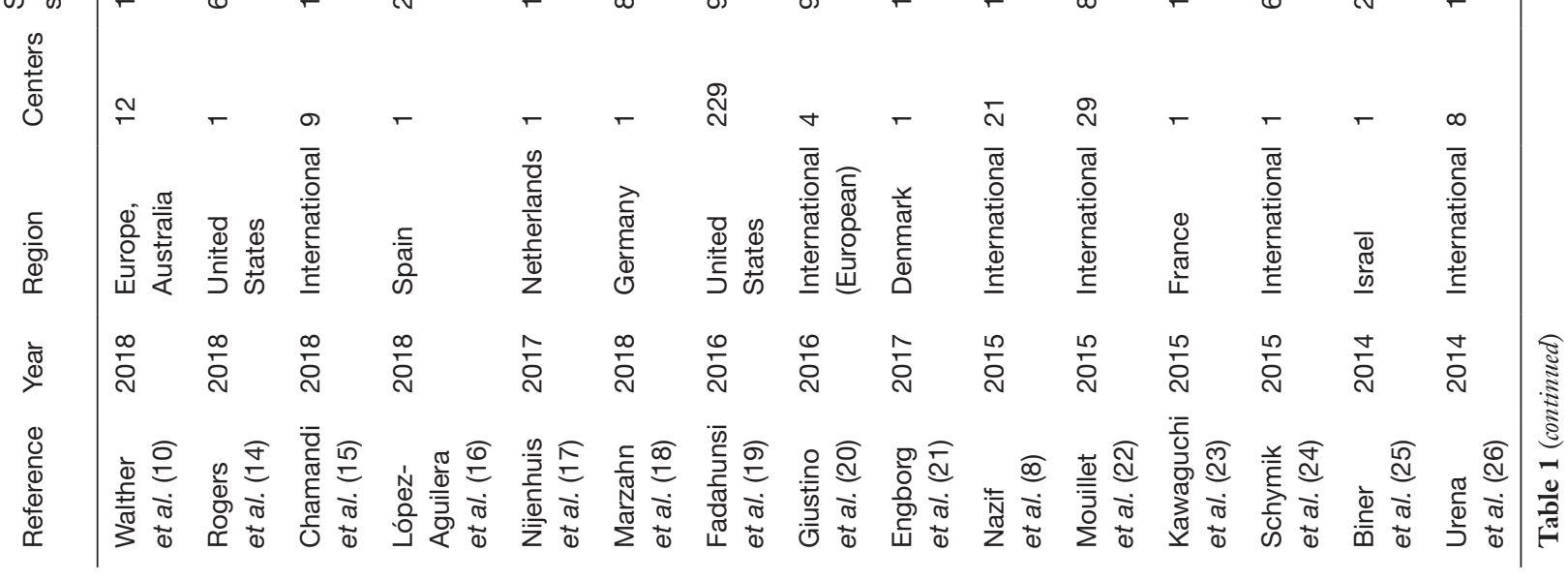




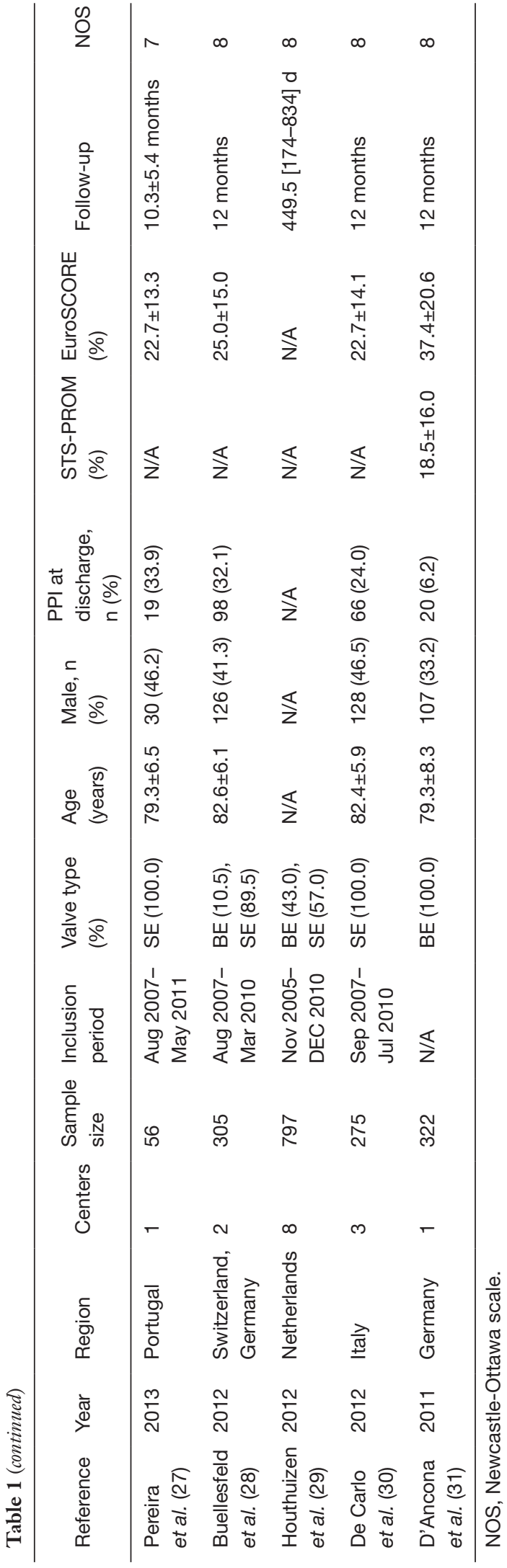

\section{Impact of permanent pacemaker implantation on the primary endpoint}

All included studies were published from June 2011 to February 2018. A PPM was implanted in 2,713 patients $(12.5 \%)$, and the rate of PPM implantation ranged from $6.2 \%$ to $32.8 \%$ among different studies. The overall incidence of all-cause death was $20.6 \%$.

There were 648 of 2,713 (23.9\%) cases of all-cause death in the PPM group compared with 3,806 of 18,953 (20.1\%) in the non-PPM group. The pooled results demonstrated a higher risk of death for patients with PPM implantation following TAVR than for those without PPM implantation following TAVR (RR, 1.13; 95\% CI, 1.01-1.25; P=0.03) (Figure 2). There was no significant heterogeneity across studies $\left(\mathrm{I}^{2}=35 \% ; \mathrm{Q}=29.08, \mathrm{P}=0.06\right)$. The pooled risk did not significantly vary when studies with a sample size of $<200$ patients were omitted (RR, 1.122; 95\% CI, 1.04-1.21; $\mathrm{P}=0.002$ ).

Possible publication bias was suggested by visual analysis of asymmetric funnel plots for the primary analysis. However, this publication bias was not confirmed by Egger's test $(\mathrm{P}=0.30)$. We also performed a correction for publication bias using the trim-and-fill method; the addition of six missing studies reduced the RR to 1.15 (95\% CI, 1.07-1.22) in the fixed-effects model and 1.20 (95\% CI, 1.03-1.38) in the random-effects model. Sensitivity analysis for the risk of all-cause death indicated that none of the studies significantly affected the results of this metaanalysis.

\section{Impact of permanent pacemaker implantation on the secondary endpoints}

The impact of PPM implantation on the risk of cardiovascular death after TAVR was evaluated by analyzing data from seven studies that included 7,407 patients. The rate of cardiovascular death was $16.8 \%(\mathrm{n}=1,245)$. Analysis of the pooled results from five studies showed that the implantation of PPM following TAVR was not associated with increased risk of cardiovascular death (RR, 0.93; 95\% CI, 0.79-1.09; $\mathrm{P}=0.37$ ) (Figure S1). There was no significant heterogeneity between studies ( $\left.\mathrm{I}^{2}=9 \% ; \mathrm{Q}=6.61, \mathrm{P}=0.36\right)$.

There were 203 of $1,464(11.8 \%)$ cases of heart failure in patients with a PPM compared with 2,682 of 12,339 (21.7\%) cases of heart failure in patients without a PPM. The incidence of heart failure did not significantly differ between patients with or without a PPM (RR, 0.90; 95\% 


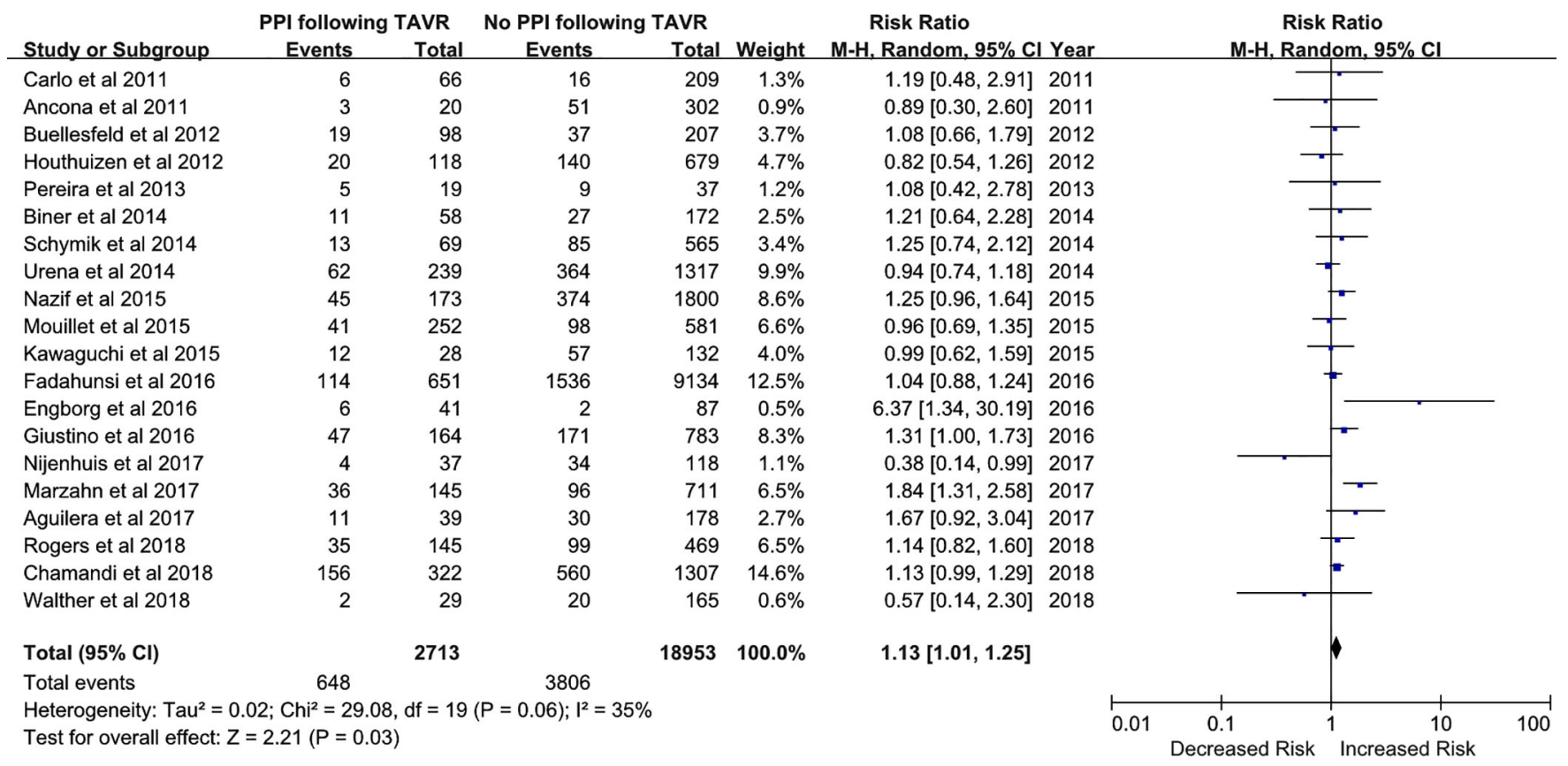

Figure 2 Risk of all-cause death in patients with new permanent pacemaker (PPM) implantation after transcatheter aortic valve replacement (TAVR).

CI, 0.50-1.62; P=0.73) (Figure S2).

Three studies provided sufficient data on the occurrence of stroke and MI during follow-up. Patients implanted with a PPM after TAVR tended to have a decreased incidence of stroke and MI compared with those without a PPM (RR, 0.85; 95\% CI, 0.64-1.13; $\mathrm{P}=0.27$ ) (Figure S3), but this difference did not reach statistical significance; this lack of significance may be due to the limited number of included studies.

\section{Incidence of permanent pacemaker implantation following transcatheter aortic valve replacement with different valve types}

A self-expandable prosthesis was implanted in 6,753 $(31.2 \%)$ patients, while a balloon-expandable prosthesis was implanted in 14,913 (68.8\%) patients. Of the 20 studies, 12 provided sufficient details on the incidence of PPM implantation in patients with implanted self-expandable or balloon-expandable prostheses. Based on data derived from these 12 studies ( $n=17,592$ patients), the implantation of a self-expandable prosthesis was associated with a 2.7fold increased risk of PPM implantation following TAVR compared with a balloon-expandable prosthesis $(25.7 \%$ vs. 6.3\%, RR, 2.77; 95\% CI, 1.95-3.93; $\mathrm{P}<0.00001$ ) (Figure 3).
There was significant heterogeneity across studies $\left(\mathrm{I}^{2}=91 \%\right.$; $\mathrm{Q}=126.67, \mathrm{P}<0.00001)$, and so a random-effects metaanalysis was performed.

\section{Discussion}

The present meta-analysis of the data from 21,666 patients included in 20 studies showed that PPM implantation following TAVR was associated with increased all-cause mortality and a tendency toward a decreased incidence of stroke and MI, but the risk of cardiovascular death did not significantly differ between patients with or without PPM implantation.

One of the most common complications after TAVR is the presence of conduction abnormalities that require the implantation of a PPM (32). Among the studies included in our meta-analysis, the rate of PPM implantation after TAVR ranged from $6.7 \%$ to $32.8 \%$. Consistent with prior studies, our results showed that patients with an implanted self-expandable prosthesis were at a significantly higher risk of new PPM implantation than those implanted with a balloon-expandable prosthesis. The fact that conduction abnormalities were more common after self-expandable versus balloon-expandable prosthesis implantation might be attributed to the valve design and the potential for 


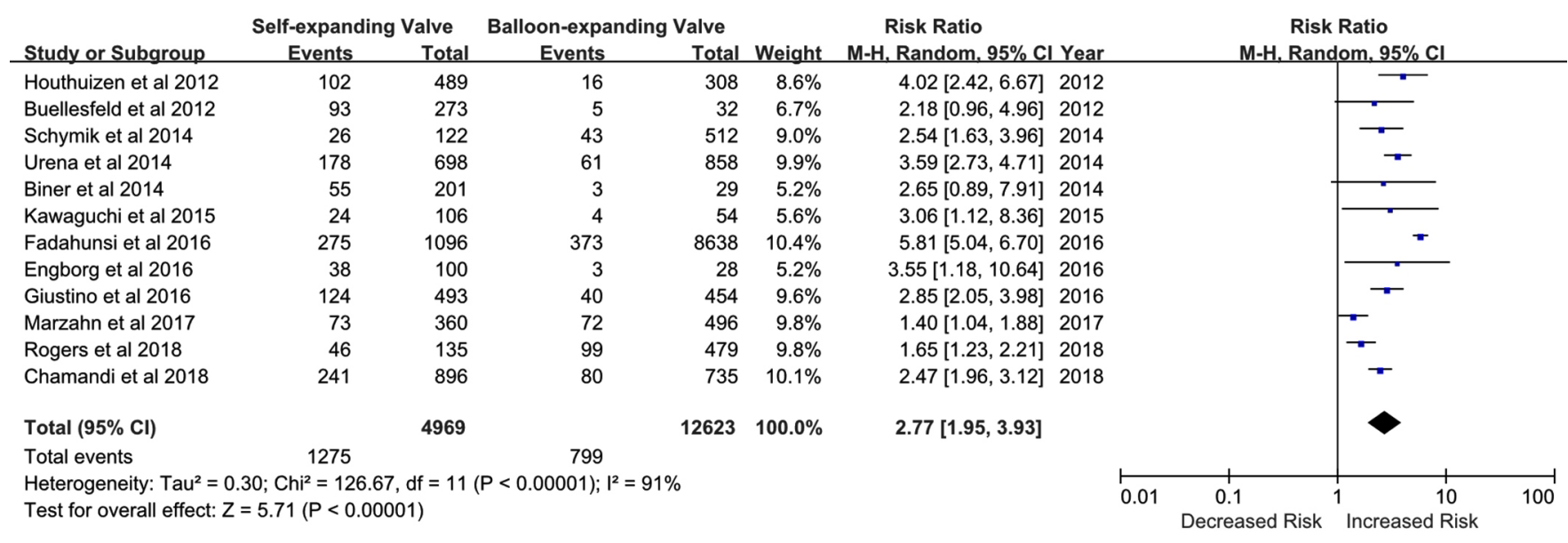

Figure 3 Incidence of permanent pacemaker (PPM) implantation after transcatheter aortic valve replacement (TAVR) with each valve type.

deeper implantation into the left ventricular outflow tract (33). For patients with a balloon-expandable prosthesis, the rate of PPM implantation remained low, regardless of whether an early- or new-generation device was used (7). However, the rate of PPM implantation remained much higher for patients with a self-expandable prosthesis, despite a reduction in the rate of PPM implantation with a new-generation device compared with an earlygeneration device. The incidence of PPM implantation may slowly decrease over time due to further technological development of TAVR and the expansion of the indications for TAVR towards lower risk patents who are younger and have less comorbidities (3).

Prior studies confirmed that advanced atrioventricular block was the most frequent reason for PPM implantation, with most pacemakers implanted within 72 hours after the TAVR procedure (15). The pathophysiological mechanism of the high incidence of atrioventricular block is the spatial proximity between the aortic valve complex and the pathway of the atrioventricular conduction system. Expansion of the prosthesis frame can result in direct trauma, compression and subsequent inflammation, hemorrhage, and ischemic or infarction injury of the conduction system, potentially causing high-grade or complete atrioventricular block (34).

Previous studies have identified several clinical and procedural factors that could predict the need for PPM implantation after TAVR. A previous metaanalysis of data from 41 studies that included 11,210 patients who underwent TAVR suggested that male sex, baseline conduction disturbances, and intraprocedural atrioventricular block are predictors of PPM implantation after TAVR with any type of prosthesis (9). Male patients tend to have more comorbidities and higher procedural risk than females, which might result in an increased risk of atrioventricular block. Pre-existing conduction abnormalities including atrioventricular block, right BBB and left BBB have been confirmed to be risk factors for postprocedural PPM implantation in numerous studies $(8,33,35,36)$. The presence and distribution of calcification underneath the aortic annulus plane and affecting the interventricular septum are anatomical factors associated with the need for PPM implantation $(8,33,37,38)$. As discussed previously, implantation depth into the left ventricular outflow tract is also strongly associated with increased risk of PPM implantation after TAVR, regardless of valve type (39).

The impact of new PPM implantation after TAVR on the long-term outcomes has been controversial in recent years. It has been proved that the need for a paced rhythm would increase the risk of late mortality and heart failure by previous evidence while the impact of paced rhythm after was still unclear $(40,41)$. The present metaanalysis found an association between PPM implantation following TAVR and an increased risk of all-cause death, but failed to show an increased risk of cardiovascular death in patients receiving new PPM implantation. Similarly, the subgroup study from the Society of Thoracic Surgeons Transcatheter Valve Therapy registry, which was included in our meta-analysis and had the largest sample size of all the included studies, found an increase in late mortality, but not cardiovascular mortality, in patients requiring PPM implantation after TAVR (19). This previous study also 
demonstrated that early PPM implantation was associated with longer median hospital stay and intensive care unit stay (19). A smaller study of 1,973 patients from the Placement of Aortic Transcatheter Valves (PARTNER) trial noted a tendency toward increased 1-year mortality in patients with new PPM implantation, although this difference did not reach statistical significance (8). These findings were in agreeance with another study in which the patients all underwent TAVR with a self-expandable prosthesis (22).

More recently, a study showed that although patients with PPM implantation after TAVR did not have an increased risk of mortality, they did have an increased risk of heart failure hospitalization (15); this study had the longest followup (median 4 years) among all the studies included in the present meta-analysis. In contrast, our meta-analysis found no significant effect of PPM implantation on the risk of heart failure. The results of studies investigating whether new PPM implantation after TAVR was associated with an increased risk of heart failure remain discordant. The following reasons might potentially explain the absence of negative outcomes in patients with PPM implantation after TAVR. Patients who had undergone TAVR were always older and had more comorbidities than patients implanted with a PPM who had not undergone TAVR, and thus the adverse effects of PPM implantation might be mitigated and not be readily apparent in the TAVR population, as the patients might not live long enough for the negative effects to develop. It is necessary to investigate the impact of PPM implantation on long-term outcomes before the indications for TAVR are expanded to include lower risk populations, as the follow-up duration in most included studies was not long enough to show the deleterious effects of PPM implantation. In addition, more than half of the patients implanted with a PPM after TAVR were not pacemaker dependent, while pacemaker dependency is a predictor of heart failure (42).

\section{Limitations}

The present meta-analysis was based on 20 non-randomized studies, and had some limitations. First, there was heterogeneity between included studies, as our meta-analysis included large multicenter registries and also single-center studies with relatively small samples. The heterogeneity among studies was also increased by the variations in endpoint definitions and inclusion criteria. Second, our meta-analysis aimed to investigate the impact of PPM implantation on long-term clinical outcomes; however, the follow-up period varied hugely among the included studies. Some included studies only followed up patients for 10 months, while others followed up patients for more than 4 years. Third, we did not exclude studies with a small sample size $(<200)$. Fourth, this was a study-level metaanalysis. An analysis of individual patient data may provide further insights.

\section{Conclusions}

The present meta-analysis provides evidence for the association between new PPM implantation after TAVR and an increased risk of all-cause death. However, new PPM implantation has no significant effect on cardiovascular mortality or the risk of MI and stroke. Moreover, the need for PPM implantation was more frequent in patients implanted with a self-expandable prosthesis compared with a ball-expandable prosthesis. Interventions to prevent conduction abnormalities and reduce the need for PPM implantation are needed before the indications of TAVR are expanded to include lower risk patients, as well as further developments regarding prosthesis design and technical features.

\section{Acknowledgments}

We thank Kelly Zammit, BVSc, from Liwen Bianji, Edanz Editing China (www.liwenbianji.cn/ac), for editing the English text of a draft of this manuscript.

\section{Footnote}

Conflicts of Interest: The authors have no conflicts of interest to declare.

Etbical Statement: The authors are accountable for all aspects of the work in ensuring that questions related to the accuracy or integrity of any part of the work are appropriately investigated and resolved.

\section{References}

1. Leon MB, Smith CR, Mack M, et al. Transcatheter aorticvalve implantation for aortic stenosis in patients who cannot undergo surgery. N Engl J Med 2010;363:1597-607.

2. Smith CR, Leon MB, Mack MJ, et al. Transcatheter versus surgical aortic-valve replacement in high-risk patients. $\mathrm{N}$ Engl J Med 2011;364:2187-98. 
3. Sondergaard L. Time to Explore Transcatheter Aortic Valve Replacement in Younger, Low-Risk Patients. JACC Cardiovasc Interv 2016;9:2183-5.

4. Khan AR, Khan S, Riaz H, et al. Efficacy and safety of transcatheter aortic valve replacement in intermediate surgical risk patients: A systematic review and metaanalysis. Catheter Cardiovasc Interv 2016;88:934-44.

5. Thourani VH, Kodali S, Makkar RR, et al. Transcatheter aortic valve replacement versus surgical valve replacement in intermediate-risk patients: a propensity score analysis. Lancet 2016;387:2218-25.

6. Chamandi C, Puri R, Rodriguez-Gabella T, et al. LatestGeneration Transcatheter Aortic Valve Replacement Devices and Procedures. Can J Cardiol 2017;33:1082-90.

7. van Rosendael PJ, Delgado V, Bax JJ. Pacemaker implantation rate after transcatheter aortic valve implantation with early and new-generation devices: a systematic review. Eur Heart J 2018;39:2003-13.

8. Nazif TM, Dizon JM, Hahn RT, et al. Predictors and clinical outcomes of permanent pacemaker implantation after transcatheter aortic valve replacement: the PARTNER (Placement of AoRtic TraNscathetER Valves) trial and registry. JACC Cardiovasc Interv 2015;8:60-9.

9. Siontis GC, Juni P, Pilgrim T, et al. Predictors of permanent pacemaker implantation in patients with severe aortic stenosis undergoing TAVR: a meta-analysis. J Am Coll Cardiol 2014;64:129-40.

10. Walther T, Manoharan G, Linke A, et al. Incidence of new-onset left bundle branch block and predictors of new permanent pacemaker following transcatheter aortic valve replacement with the Portico valve. Eur J Cardiothorac Surg 2018;54:467-74.

11. Regueiro A, Abdul-Jawad Altisent O, Del Trigo M, et al. Impact of New-Onset Left Bundle Branch Block and Periprocedural Permanent Pacemaker Implantation on Clinical Outcomes in Patients Undergoing Transcatheter Aortic Valve Replacement: A Systematic Review and MetaAnalysis. Circ Cardiovasc Interv 2016;9:e003635.

12. Moher D, Liberati A, Tetzlaff J, Altman DG. Preferred reporting items for systematic reviews and metaanalyses: the PRISMA statement. Ann Intern Med 2009;151:264-9, w64.

13. Stang A. Critical evaluation of the Newcastle-Ottawa scale for the assessment of the quality of nonrandomized studies in meta-analyses. Eur J Epidemiol 2010;25:603-5.

14. Rogers T, Devraj M, Thomaides A, et al. Utility of Invasive Electrophysiology Studies in Patients With Severe Aortic Stenosis Undergoing Transcatheter Aortic Valve
Implantation. Am J Cardiol 2018;121:1351-7.

15. Chamandi C, Barbanti M, Munoz-Garcia A, et al. LongTerm Outcomes in Patients With New Permanent Pacemaker Implantation Following Transcatheter Aortic Valve Replacement. JACC Cardiovasc Interv 2018;11:301-10.

16. López-Aguilera J, Segura Saint-Gerons JM, Sanchez Fernandez J, et al. Long-term clinical impact of permanent cardiac pacing after transcatheter aortic valve implantation with the CoreValve prosthesis: a single center experience. Europace 2018;20:993-1000.

17. Nijenhuis VJ, Van Dijk VF, Chaldoupi SM, et al. Severe conduction defects requiring permanent pacemaker implantation in patients with a new-onset left bundle branch block after transcatheter aortic valve implantation. Europace 2017;19:1015-21.

18. Marzahn C, Koban C, Seifert M, et al. Conduction recovery and avoidance of permanent pacing after transcatheter aortic valve implantation. J Cardiol 2018;71:101-8.

19. Fadahunsi OO, Olowoyeye A, Ukaigwe A, et al. Incidence, Predictors, and Outcomes of Permanent Pacemaker Implantation Following Transcatheter Aortic Valve Replacement: Analysis From the U.S. Society of Thoracic Surgeons/American College of Cardiology TVT Registry. JACC Cardiovasc Interv 2016;9:2189-99.

20. Giustino G, Van der Boon RM, Molina-Martin de Nicolas $\mathrm{J}$, et al. Impact of permanent pacemaker on mortality after transcatheter aortic valve implantation: the PRAGMATIC (Pooled Rotterdam-Milan-Toulouse in Collaboration) Pacemaker substudy. EuroIntervention 2016;12:1185-93.

21. Engborg J, Riechel-Sarup C, Gerke O, et al. Effect of permanent pacemaker on mortality after transcatheter aortic valve replacement. Scand Cardiovasc J 2017;51:40-6.

22. Mouillet G, Lellouche N, Yamamoto M, et al. Outcomes following pacemaker implantation after transcatheter aortic valve implantation with CoreValve((R)) devices: Results from the FRANCE 2 Registry. Catheter Cardiovasc Interv 2015;86:E158-66.

23. Kawaguchi AT, D'Allessandro C, Collet JP, et al. Ventricular conduction defects after transcatheter aortic valve implantation: a single-institute analysis. Artif Organs 2015;39:409-15.

24. Schymik G, Tzamalis P, Bramlage P, et al. Clinical impact of a new left bundle branch block following TAVI implantation: 1-year results of the TAVIK cohort. Clin Res Cardiol 2015;104:351-62. 
25. Biner S, Michowitz Y, Leshem-Rubinow E, et al. Hemodynamic impact and outcome of permanent pacemaker implantation following transcatheter aortic valve implantation. Am J Cardiol 2014;113:132-7.

26. Urena M, Webb JG, Tamburino C, et al. Permanent pacemaker implantation after transcatheter aortic valve implantation: impact on late clinical outcomes and left ventricular function. Circulation 2014;129:1233-43.

27. Pereira E, Ferreira N, Caeiro D, et al. Transcatheter aortic valve implantation and requirements of pacing over time. Pacing Clin Electrophysiol 2013;36:559-69.

28. Buellesfeld L, Stortecky S, Heg D, et al. Impact of permanent pacemaker implantation on clinical outcome among patients undergoing transcatheter aortic valve implantation. J Am Coll Cardiol 2012;60:493-501.

29. Houthuizen P, Van Garsse LA, Poels TT, et al. Left bundle-branch block induced by transcatheter aortic valve implantation increases risk of death. Circulation 2012;126:720-8.

30. De Carlo M, Giannini C, Bedogni F, et al. Safety of a conservative strategy of permanent pacemaker implantation after transcatheter aortic CoreValve implantation. Am Heart J 2012;163:492-9.

31. D'Ancona G, Pasic M, Unbehaun A, et al. Permanent pacemaker implantation after transapical transcatheter aortic valve implantation. Interact Cardiovasc Thorac Surg 2011;13:373-6.

32. Urena M, Rodes-Cabau J. Conduction Abnormalities: The True Achilles' Heel of Transcatheter Aortic Valve Replacement? JACC Cardiovasc Interv 2016;9:2217-9.

33. Mauri V, Reimann A, Stern D, et al. Predictors of Permanent Pacemaker Implantation After Transcatheter Aortic Valve Replacement With the SAPIEN 3. JACC Cardiovasc Interv 2016;9:2200-9.

34. van der Boon RM, Nuis RJ, Van Mieghem NM, et al.

Cite this article as: Xi Z, Liu T, Liang J, Zhou YJ, Liu W. Impact of postprocedural permanent pacemaker implantation on clinical outcomes after transcatheter aortic valve replacement: a systematic review and meta-analysis. J Thorac Dis 2019;11(12):5130-5139. doi: 10.21037/jtd.2019.12.02
New conduction abnormalities after TAVI--frequency and causes. Nat Rev Cardiol 2012;9:454-63.

35. Steinberg BA, Harrison JK, Frazier-Mills C, et al. Cardiac conduction system disease after transcatheter aortic valve replacement. Am Heart J 2012;164:664-71.

36. Keßler M, Gonska B, Seeger J, et al. Predictors of permanent pacemaker implantation after transfemoral aortic valve implantation with the Lotus valve. Am Heart J 2017;192:57-63.

37. Fujita B, Kutting M, Seiffert M, et al. Calcium distribution patterns of the aortic valve as a risk factor for the need of permanent pacemaker implantation after transcatheter aortic valve implantation. Eur Heart J Cardiovasc Imaging 2016;17:1385-93.

38. Maeno Y, Abramowitz Y, Kawamori H, et al. A Highly Predictive Risk Model for Pacemaker Implantation After TAVR. JACC Cardiovasc Imaging 2017;10:1139-47.

39. Almeida JG, Ferreira SM, Fonseca P, et al. Association between implantation depth assessed by computed tomography and new-onset conduction disturbances after transcatheter aortic valve implantation. J Cardiovasc Comput Tomogr 2017;11:332-7.

40. Freudenberger RS, Wilson AC, Lawrence-Nelson J, et al. Permanent pacing is a risk factor for the development of heart failure. Am J Cardiol 2005;95:671-4.

41. Dewland TA, Pellegrini CN, Wang Y, et al. Dual-chamber implantable cardioverter-defibrillator selection is associated with increased complication rates and mortality among patients enrolled in the NCDR implantable cardioverterdefibrillator registry. J Am Coll Cardiol 2011;58:1007-13.

42. Alasti M, Rashid H, Rangasamy K, et al. Longterm pacemaker dependency and impact of pacing on mortality following transcatheter aortic valve replacement with the LOTUS valve. Catheter Cardiovasc Interv 2018;92:777-82. 


\section{Supplementary}

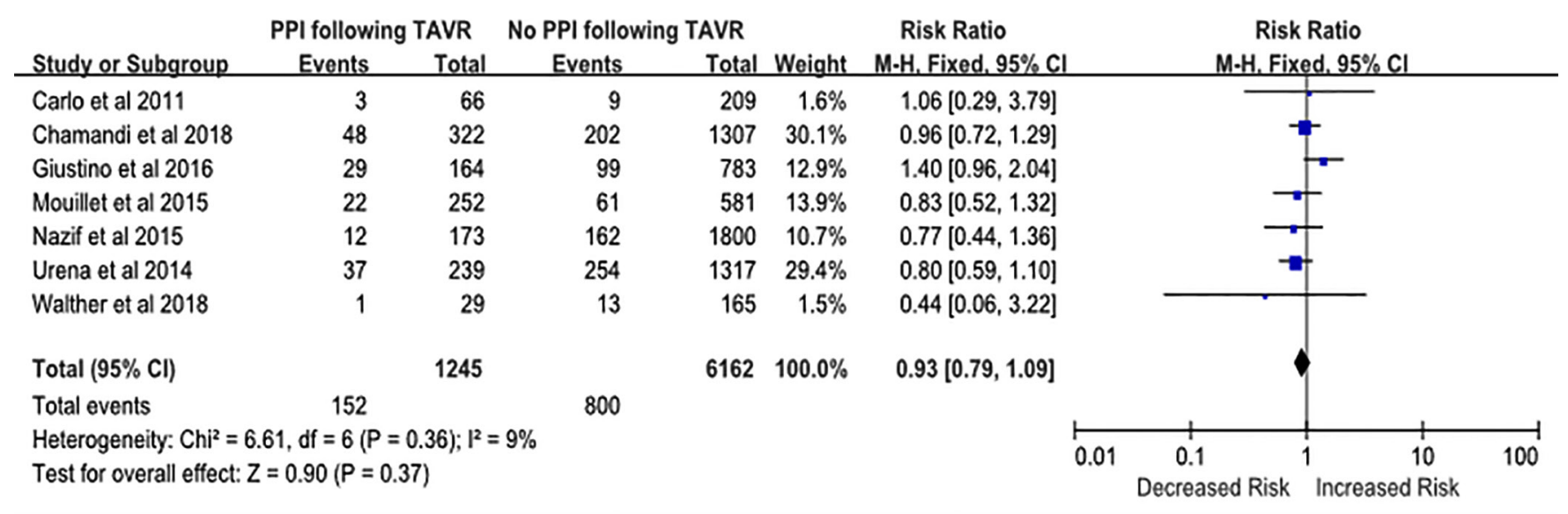

Figure S1 Risk of cardiovascular death in patients with new permanent pacemaker (PPM) implantation after transcatheter aortic valve replacement (TAVR).

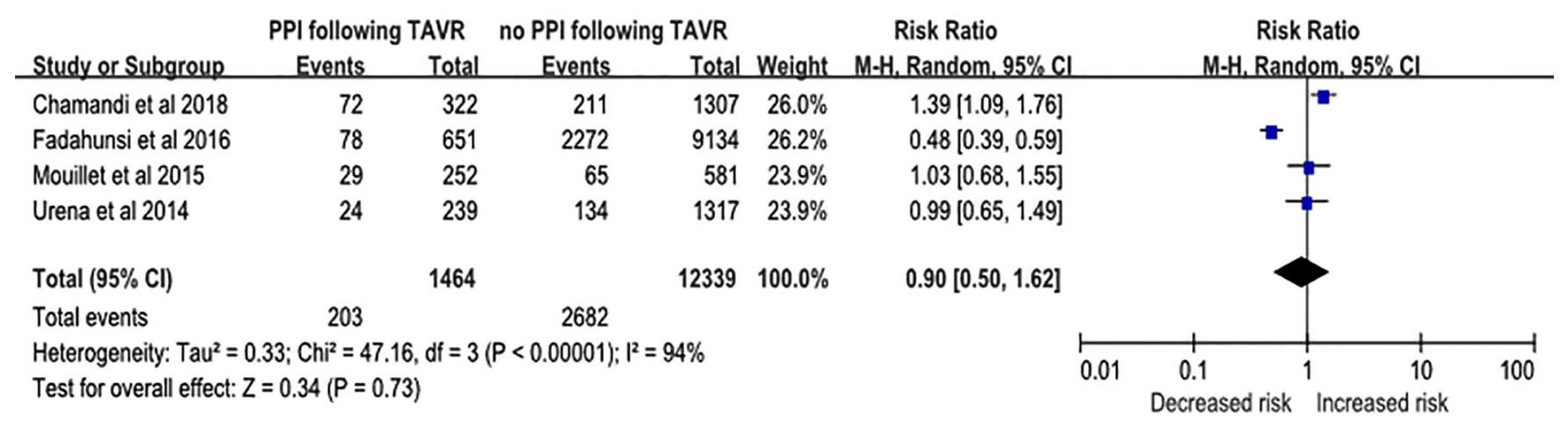

Figure S2 Risk of heart failure in patients with new permanent pacemaker (PPM) implantation after transcatheter aortic valve replacement (TAVR).

Experimental Control Risk Ratio

Study or Subgroup Events Total Events Total Weight M.H. Fixed, 95\% Cl

\begin{tabular}{|c|c|c|c|c|c|c|}
\hline Buellesfeld et al 2012 & 3 & 98 & 13 & 207 & $8.1 \%$ & $0.49[0.14,1.67]$ \\
\hline Fadahunsi et al 2016 & 36 & 651 & 532 & 9134 & $68.7 \%$ & $0.95[0.68,1.32]$ \\
\hline Nazif et al 2015 & 9 & 173 & 136 & 1800 & $23.2 \%$ & $0.69[0.36,1.33]$ \\
\hline Total $(95 \% \mathrm{Cl})$ & & 922 & & 11141 & $100.0 \%$ & $0.85[0.64,1.13]$ \\
\hline Total events & 48 & & 681 & & & \\
\hline \multicolumn{7}{|c|}{ Heterogeneity: $\mathrm{Ch}^{2}=1.61, \mathrm{df}=2(\mathrm{P}=0.45) ; 1^{2}=0 \%$} \\
\hline \multicolumn{7}{|c|}{ Test for overall effect: $Z=1.11(P=0.27)$} \\
\hline
\end{tabular}

\section{Risk Ratio}

\section{M.H. Fixed. $95 \% \mathrm{Cl}$}

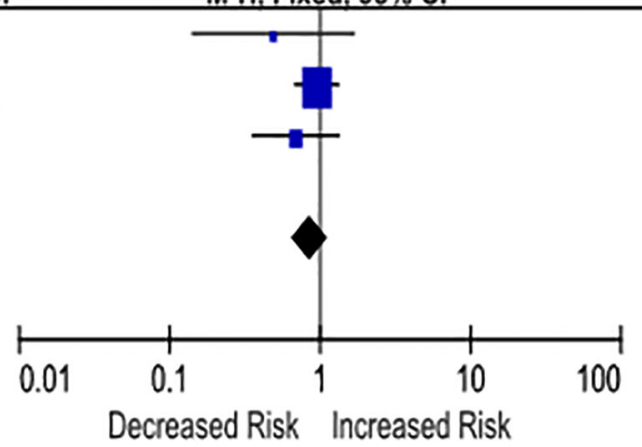

Figure S3 Risk of myocardial infarction (MI) and stroke in patients with new permanent pacemaker (PPM) implantation after transcatheter aortic valve replacement (TAVR). 